\title{
Lidil
}

Revue de linguistique et de didactique des langues

47 | 2013

Le verbe pour exprimer le temps

\section{Louise Dabène : un itinéraire hors du commun}

HOMMAGE

Jacqueline Billiez, Christian Degache et Diana-Lee Simon

\section{CpenEdition}

Journals

Édition électronique

URL : http://journals.openedition.org/lidil/3259

DOI : 10.4000/lidil.3259

ISSN : 1960-6052

Éditeur

UGA Éditions/Université Grenoble Alpes

\section{Édition imprimée}

Date de publication : 31 mai 2013

Pagination : 5-13

ISBN : 978-2-84310-247-9

ISSN : $1146-6480$

Référence électronique

Jacqueline Billiez, Christian Degache et Diana-Lee Simon, « Louise Dabène : un itinéraire hors du commun », Lidil [En ligne], 47 | 2013, mis en ligne le 31 mai 2013, consulté le 15 septembre 2020. URL http://journals.openedition.org/lidil/3259

(c) Lidil 


\title{
Louise Dabène : un itinéraire hors du commun ${ }^{1}$
}

\author{
Jacqueline Billiez, Christian Degache et Diana-Lee Simon*
}

\begin{abstract}
admirable, amicale; brillante, bilingue; chaleureuse, conviviale, claire, curieuse; dynamique, drôle, dévouée; énergique, exubérante; forte ; gaie; humaine; habile; initiatrice, inventive, infatigable; jeune, joviale ; kamikaze; lucide; mobile; multilingue; novatrice; ouverte, obstinée; perspicace, pétillante, prolifique; quiète; rapide, réfléchie; stimulante, sympathique, synthétique ; trépidante, travailleuse ; unique, vive, vivante; Wallah; xénophile; synalephe, zélatrice.
\end{abstract}

Il fallait bien un «Abécédaire de Louise ${ }^{2}$ » avec ses entrées multiples par lettre pour capter, ne serait-ce que fugitivement, les qualités, les traits de personnalité et les dimensions multiples évocatrices d'une femme, d'une collègue, d'une amie, d'une enseignante, d'une chercheuse, d'une collaboratrice, qui aura marqué ceux et celles qui l'ont côtoyée, connue croisée ou lue sur le chemin de la didactique des langues et du plurilinguisme. Offert en hommage à l'occasion de sa retraite en 1998, cet assemblage lexical frivole prend aujourd'hui une nouvelle tonalité affective au moment même où nous cherchons à créer un espace de recueillement, grave et digne de notre chère Louise, disparue à notre grande tristesse le 8 avril 2013.

Suivons le parcours de Louise...

* Respectivement professeure émerite, professeur des universités et maitre de conférence, Université Grenoble Alpes, LIDILEM, F-38040 Grenoble.

1. Nous souhaitons exprimer ici notre profonde reconnaissance à Michel Dabène qui a mis à notre disposition un document très précieux de référence sous forme de note, établie par Louise Dabène elle-même, pour l'obtention de l'éméritat.

2. Foerster, Simon \& Galligani. (1998). Abécédaire de Louise. Dans Billiez (dir.), De la didactique des langues à la didactique du plurilinguisme. Hommage à Louise Dabène (p. 9-16). CDL-LIDILEM, Université Stendhal-Grenoble 3. 


\section{Itinéraire biographique et assise du champ didactique}

Une solide formation entre 1954 et 1958, en tant qu'élève-professeur à L'École normale supérieure de Fontenay-aux-Roses ${ }^{3}$, prépare Louise Dabène pour ses fonctions de professeure agrégée d'espagnol, qu'elle exercera au lycée pilote de Sèvres, entre 1958 et 1967. Passionnée de l'innovation méthodologique dans l'enseignement des langues, elle y met déjà en œuvre une approche communicative et audio-visuelle novatrice $^{4}$ et ne tarde pas à établir des liens avec d'autres jeunes professeurs, comme Danièle Bailly, enseignante d'anglais dans le même établissement, donnant lieu à la création d'un petit «Groupe d'études de langues» où règne une ambiance conviviale et productive autour de la recherche et des échanges.

Après l'obtention en 1967 de sa thèse, un doctorat ès Lettres et sciences humaines en linguistique hispanique, elle exerce comme assistante d'espagnol pendant deux ans à l'université de Nanterre, avant d'obtenir en 1969 un poste de maitre-assistante à l'Institut de linguistique appliquée et de didactique des langues de l'université de Vincennes, une nouvelle université dont elle est membre du collectif fondateur.

À partir de 1973, elle s'installe à Grenoble pour occuper à l'université Stendhal un poste de maitre de conférences puis de professeure des universités. Les vingt-cinq ans de carrière suivants seront marquants, tant du point de vue des créations institutionnelles qui ont donné une visibilité et une légitimité scientifique à la didactique des langues et fortement contribué à l'émergence et à la reconnaissance du champ comme domaine à part entière, que des initiatives multiples au niveau de l'innovation dans le domaine de la recherche en sociolinguistique et didactique des langues. On notera ici sa création en 1974 du Centre de didactique des langues (CDL), et celle du Centre de didactique de français (CDF) à l'initiative de Michel Dabène, assurant ainsi une assise institutionnelle à cette jeune discipline didactique en quête de légitimation universitaire. Elle est fondatrice en 1987, avec Michel Dabène, du laboratoire Lidilem (Linguistique et didactique des langues étrangères et maternelles), qu'elle dirigera jusqu'en 1998, marquant ainsi

3. Aujourd'hui École normale supérieure des Lettres et Sciences humaines (ENS LSH de Lyon).

4. Elle est auteure de plusieurs manuels d'espagnol, dont ¿Qué tal Carmen? (1968) et ¡Tú también! (1972). 
une étape décisive pour la reconnaissance et le prestige internationaux non seulement des recherches en didactique des langues, mais aussi de celles en linguistique et sociolinguistique comme en témoignent encore aujourd'hui la vitalité et la taille de ce laboratoire.

Comme support à la publication et à la diffusion de ces recherches auprès de la communauté des chercheurs, la revue Lidil voit le jour en 1989 (date de publication des deux premiers numéros), sous l'impulsion de Louise Dabène, qui devient rédactrice en chef, et le restera jusqu'en 1998 .

Une anecdote permettra de saisir au vol quelques dimensions charismatiques et caractéristiques d'une Louise, chef d'équipe, menant le jeu. Nous sommes fin 1994. Oui, votre calcul est bon! Il s'agit des vingt ans du CDL, et cela se fête selon la tradition de convivialité instaurée par Louise. On se souvient encore de cette occasion festive, à la fois mythique et emblématique, lors de laquelle Louise, déguisée en capitaine, a fait irruption dans la grande salle des colloques, suivie de son équipe d'une vingtaine de chercheurs du CDL, tous en tenue de rugby, se passant habilement le ballon, avec Denis Creissels, déguisé en arbitre et donnant des coups de sifflet!

Tout n'était pourtant pas toujours «de la tarte», pour ainsi dire, et certaines batailles nécessitaient un courage à toute épreuve. Dynamique et dévouée, Louise Dabène ne manquera pas de relever le défi de fédérer les associations françaises de linguistique appliquée pour assurer une présence française au niveau mondial de l'Association internationale de linguistique appliquée (AILA), en créant la Confédération française de linguistique appliquée (COFDELA) en 1994, avec un premier symposium qui s'est tenu à Grenoble en 1996, et un deuxième à Strasbourg en 1997. Infatigable avec une puissance de travail hors du commun, c'est à cette époque qu'elle occupe la fonction de vice-présidente à la recherche à l'université Stendhal.

Par ailleurs, ses initiatives en faveur de la didactique des langues concernent également la vie associative. Créée dès 1987, grâce aux efforts concertés d'une petite équipe d'aficionados autour de Louise Dabène, l'ACEDLE (Association des chercheurs et enseignants didacticiens de langues étrangères $)^{5}$, comme son nom l'indique, offre un espace d'échanges, de dialogue et de réflexion scientifiques sur la didactique de différentes langues, ouvert aux enseignants et aux chercheurs,

5. L'ACEDLE, actuellement sous la présidence de Dominique Macaire, est encore très active : http://acedle.org/ 
encouragés à se rencontrer et à travailler ensemble. Les colloques et publications réguliers de l'ACEDLE ont, par ailleurs, contribué à documenter - sous la plume de Louise Dabène avec d'autres didacticiens fervents - l'évolution épistémologique du domaine ${ }^{6}$. Elle présidera l'association de 1987 à 1999.

Un itinéraire dense et engagé, certes, qui a aussi fortement marqué la recherche sur les contacts de langues en France, tant du point de vue sociolinguistique que didactique. Au moment où elle prendra sa retraite fin 1998, le champ établi et reconnu de la didactique des langues aura considérablement évolué sur le plan épistémologique vers une didactique du plurilinguisme ${ }^{7}$, et maints projets, tous originaux et novateurs, auront été lancés, dont des programmes européens de grande envergure sur l'intercompréhension entre locuteurs de langues romanes (programme Galatea en $1991^{8}$ ) et l'éveil aux langues (expériences amorcées dès 1988 dans une école de la banlieue grenobloise, puis participation au programme européen Evlang), programmes de recherche au long cours qui, à sa suite, se sont révélés pérennes, comme on le verra ci-dessous.

\section{Itinéraire de recherche : terrains et théorisations}

Tous les chercheurs et enseignants qui l'ont côtoyée se souviennent d'un principe intangible qui devait gouverner les travaux de recherche: ceux-ci devaient partir du terrain pour y revenir. Les analyses et théorisations devaient tenter de répondre aux besoins du terrain et déboucher sur des propositions didactiques en direction des différents publics d'apprenants auxquels elle s'est intéressée. En premier lieu ses élèves apprenant l'espagnol langue étrangère en contexte scolaire français pour lesquels elle a conçu, dans une perspective communicative, plusieurs manuels qui ont été utilisés, pendant une très longue période et avec grand profit, par les enseignants, qui leur reconnaissaient un caractère très novateur comme en témoigne Didier Rault (maitre de conférences,

6. Cf. Dabène, L. (2000). Pour une didactique plurielle. Quelques réflexions. Dans Billiez (dir.), La didactique des langues dans l'espace francophone : Unité et diversité (p. 9-13).

7. Cf. Billiez (dir.). (1998). De la didactique des langues à la didactique du plurilinguisme. Hommage à Louise Dabène. CDL-LIDILEM, Université Stendhal-Grenoble 3.

8. Voir http://www.galanet.eu/publication/fichiers/L_Dabene1994_EH22.pdf 
université Paul Valéry-Montpellier 3), adressant ses condoléances aux collègues du Lidilem :

J'ai appris en lisant Le Monde le décès de notre collègue Louise Dabène. Je ne la connaissais pas personnellement, mais j'ai utilisé avec grand profit les ouvrages qu'elle avait composés (¿Qué tal Carmen?, ¿Adónde?) et lu certains de ses travaux de recherche. Elle a joué un rôle important dans la rénovation de l'enseignement de l'espagnol et je souhaite donc vous exprimer ma reconnaissance envers elle et ses travaux, qui ont été des raisons d'espérer que l'enseignement des langues vivantes, et de l'espagnol tout particulièrement, pouvait être moderne, plaisant et efficace. Il reste beaucoup à faire.

Puis, dès les années 1970, Louise Dabène a développé et impulsé des analyses et des propositions didactiques en direction de publics adultes qui s'inscrivaient à l'université, dans le cadre de la formation continue, pour des cours de diverses langues - dont celles de migrants, comme l'arabe - travaux qui ont rencontré les orientations des institutions européennes. En continuité avec cet intérêt pour les langues et cultures d'origine des populations migrantes, elle s'est alors intéressée, dès les années 1980, à l'ensemble des problèmes posés, dans le contexte scolaire, par leur enseignement à leur descendance. Analysées grâce aux outils de la sociolinguistique, leurs spécificités langagières plurilingues, ainsi que celles étudiées dans de très nombreuses thèses de doctorants en provenance de diverses contrées multilingues du monde, lui permettront d'affiner les stratégies d'approche de ces phénomènes complexes et de proposer des théorisations et notions nouvelles, clairement exposées dans l'ouvrage qui sert de référence incontournable dans ce domaine, Repères sociolinguistiques pour l'enseignement des langues (1994).

Les recherches conduites par l'équipe de Louise Dabène au CDL, à la fin des années 1970 et ensuite pendant les années 1980, portant sur le développement d'activités métalinguistiques par l'apprenant confronté à l'apprentissage du français et des langues étrangères, disciplines dont elle déplorait le cloisonnement en milieu scolaire, cherchaient à éclairer la question : «Quelles sont les modalités par lesquelles se constitue la conscience métalinguistique ?» Bien au-delà de l'apprentissage d'une langue particulière, c'est l'éducation langagière au sens plus large qui l'intéressait, et surtout des modalités d'apprentissage susceptibles d'aider les enfants, tous, à établir des passerelles entre leurs langues familiales et scolaires et à dépasser leur chauvinisme linguistique. Dans cette optique, et en s'appuyant sur les recherches issues du 
courant «Language Awareness » impulsé en Angleterre par Eric Hawkins (1984), se sont développées au CDL et en France sous la désignation «Éveil aux langues», de nombreux travaux et projets. Louise Dabène et son équipe se sont lancées concrètement dans la conception d'activités pédagogiques dont les premières expérimentations furent amorcées à l'école Robespierre, à Fontaine en 1988. Plusieurs articles ${ }^{9}$ publiés dans les années 1990 interrogent le curriculum scolaire sur l'enseignement d'une seule langue, en invitant à une méthodologie plurielle. Une rencontre organisée à son initiative fin 1996 à l'université Stendhal, a permis de réunir des collègues de Paris, de Suisse, d'Italie, d'Allemagne, d'Espagne, d'Autriche, travaillant dans ce sens. Elle a donné lieu à naissance d'un projet européen Socrates Lingua, intitulé Evlang (1997-2001), dont la coordination est confiée à Michel Candelier, qui continuera après à en piloter plusieurs autres, dont Janua Linguarum. À la porte des langues, et le grand projet CARAP (Cadre de référence pour les approches plurielles) en cours actuellement. La force de ces propositions, qui font de la diversité linguistique et culturelle un matériau didactique, s'étend dans plusieurs pays européens (Allemagne, Belgique, Espagne, France, Italie, Suisse) et au-delà au Canada ${ }^{10}$.

En cohérence avec cette approche, Louise Dabène pense également que l'on ne tire pas tout le parti que l'on pourrait de la parenté linguistique et qu'il est nécessaire d'en reconsidérer positivement le rôle, notamment pour l'enseignement des langues romanes. Ainsi, au début des années 1990, elle reprend cette idée qui lui était chère depuis la publication dans la revue Langages en 1975 de son article «L'enseignement de l'espagnol aux francophones (Pour une didactique des langues "voisines") ${ }^{11} \gg$ qui peut être considéré comme fondateur des travaux en didactique des langues sur l'intercompréhension. Le contexte européen est alors très favorable à la relance de ce projet qu'elle choisit d'appeler Galatea. En conformité avec le principe de va-et-vient entre le terrain et le recul théorique mentionné ci-dessus, Louise Dabène conçoit son

9. Pour une liste exhaustive des publications de Louise Dabène, voir dans Billiez (dir.) (1998), De la didactique des langues à la didactique du plurilinguisme. Hommage à Louise Dabène, CDL-LIDILEM, Université StendhalGrenoble 3, 405-410.

10. Cf. http://www.elodil.com/

11. Langages, $\mathrm{n}^{\circ} 39$, disponible sur http://www.galanet.eu/publication/fichiers/ dabene.pdf 
projet en deux phases. Une première phase d'études exploratoires préalables centrée, d'une part, sur le recueil et l'analyse des stratégies et des représentations d'échantillons représentatifs, et, d'autre part, sur l'analyse contrastive des langues voisines dans la perspective d'identifier des «règles de passage et de vigilance». Puis une deuxième phase consistant à élaborer un guidage méthodologique à travers la création d'un matériel pédagogique adapté. Cette phase se développera surtout à partir de 1995, dans le cadre du programme Lingua de la Commission européenne destiné à encourager l'apprentissage des langues, avec la réalisation de sept cédéroms Galatea publiés de 2000 à 2003. Pourquoi Galatea? La question est souvent posée. On a pu chercher à l'expliquer par un acronyme mais cela n'avait rien à voir. Louise Dabène justifiait parfois ce choix, non sans malice, par le fait que, dans la mythologie, la nymphe Galatea avait préféré l'amour d'Acis, le simple berger, à celui de Polyphème, le cyclope, fort et puissant mais dont l'œil unique pouvait être vu comme un symbole du monolinguisme. Toutefois, la raison principale était sans doute qu'elle aimait avant tout ce nom. Elle a d'ailleurs transmis cette affection à toutes celles et ceux qui ont participé au projet avec elle de janvier 1992, date de la première réunion à Grenoble, à la fin du projet européen en 1999, puis, ultérieurement, dans le cadre du projet Galanet qui en fut la continuité sur Internet et auquel elle a participé activement jusqu'en 2003, notamment pour les développements concernant la compréhension orale. On a pu voir une certaine concurrence du projet Galatea avec le projet EuRom4 lancé également au début des années 1990 par Claire Blanche-Benveniste, mais les démarches étaient sensiblement différentes et en réalité complémentaires, comme l'ont prouvé les contacts établis dès les premières années entre les deux équipes puis le rapprochement effectif en 2006 dans le projet Diálogos em intercompreensão et ensuite dans le réseau Redinter et les projets Galapro et Miriadi. Après 2003, Louise Dabène est restée informée de ces développements comme en témoigne le message qu'elle a adressé aux organisateurs du colloque Intercompréhension : compétences plurielles, corpus, intégration ${ }^{12}$ qui s'est tenu à Grenoble en juin 2012 et à l'occasion duquel un hommage lui a été rendu :

12. Voir http://ic2012.u-grenoble3.fr/ et http://podcast.grenet.fr/episode/ic-2012ouverture-21062012/ 
Je suis très touchée par l'hommage que vous avez prévu de me rendre à l'ouverture de votre colloque. Qui aurait pu penser il y a vingt ans qu'à partir d'une modeste hypothèse de travail se développeraient des recherches et des productions de documents aussi impressionnantes, mobilisant autant de compétences de plusieurs pays?

Je souhaite partager cet hommage avec toutes celles et tous ceux qui ont collaboré à cette entreprise, les anciens avec qui j'ai eu le plaisir de travailler et ceux qui ont suivi et que je ne connais que par leur nom. Il est indéniable que vos travaux apportent une contribution décisive à la didactique des langues.

À toutes et à tous je souhaite beaucoup de réussite dans la suite de vos projets.

Avec mes plus cordiales pensées, Louise Dabène. (Bidart, 5 juin 2012)

Comme le souligne justement Maria Elena Ceberio, de l'université nationale de Rio Cuarto en Argentine où Louise Dabène s'est rendue à plusieurs reprises pour y parler d'intercompréhension, «su "Galasaga" prosperó».

Pendant sa retraite, Louise Dabène, inlassable chercheure, a poursuivi des travaux personnels et surtout gardé le contact avec de nombreux collègues et anciens étudiants. Redéployant ses racines au Pays basque, elle s'est intéressée notamment à l'enseignement bilingue au niveau pré-élémentaire et élémentaire avec sa collègue et amie Miren Garmendia, conseillère pédagogique à Saint-Jean-de-Luz, tout en théorisant les options et complexités dans un de ses derniers articles intitulé : «Enseigner en langue régionale ${ }^{13}$ ». Elle n'a cependant pas résolu la question de l'origine mystérieuse de la langue basque !

Livrons enfin un dernier élément, éclairant aussi la grande humanité de Louise, relaté à ses obsèques par sa charmante auxiliaire de vie basque qui nous a confié en aparté que Louise lui faisait encore les derniers jours de sa vie un enseignement - ludique ! - de l'espagnol à l'aide de son manuel ¿Qué tal, Carmen? Elle nous a soufflé : «J'ai eu le privilège de m'occuper d'une grande dame.»

13. Cf. Dabène, L. \& Garmendia, M. (2003). Enseigner en langue régionale. Le Plurilinguisme en construction dans le système éducatif. Contextes-dispositifs-acteurs, Lidil, hors-série, 165-173. 


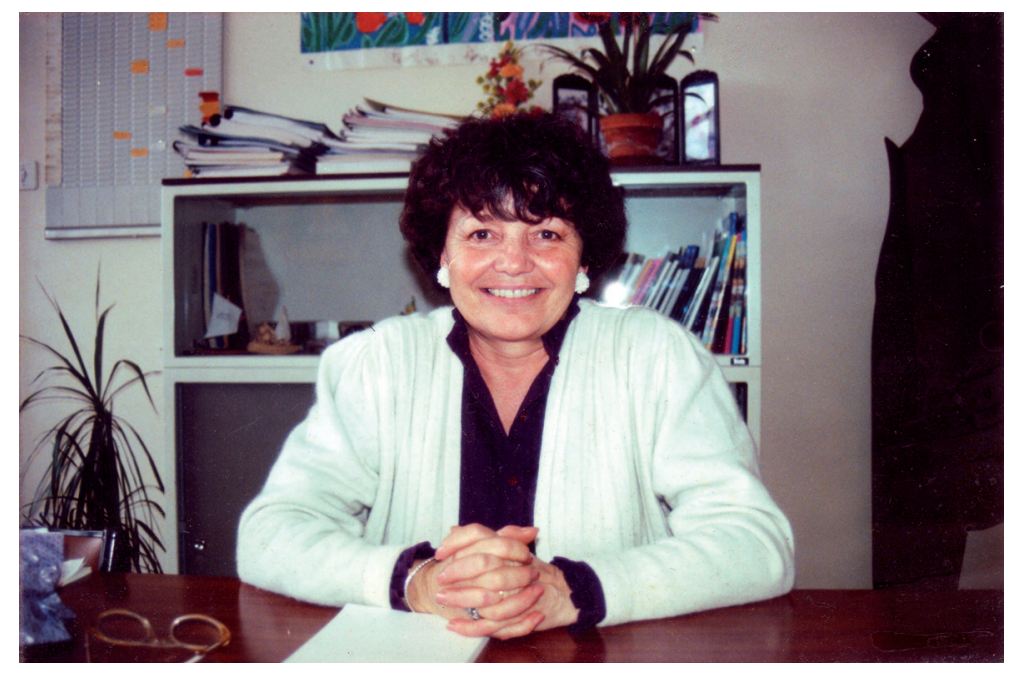

